Article

\title{
Exclusion-Zone Dynamics Explored with Microfluidics and Optical Tweezers
}

\author{
István N. Huszár ${ }^{1, \dagger}$, Zsolt Mártonfalvi ${ }^{1, \dagger}$, András József Laki ${ }^{2}$, Kristóf Iván ${ }^{2}$ and \\ Miklós Kellermayer ${ }^{1,3, *}$
}

1 Department of Biophysics and Radiation Biology, Semmelweis University, Tủzoltó utca 37-47, Budapest H1094, Hungary; E-Mails: istvan.n.huszar@gmail.com (I.N.H.); martonfalvi.zsolt@med.semmelweis-univ.hu (Z.M.)

2 Faculty of Information Technology and Bionics, Pázmány Péter University, Práter utca 50/A, Budapest H1083, Hungary; E-Mails: laki.andras@itk.ppke.hu (A.J.L.); ivan.kristof@itk.ppke.hu (K.I.)

3 MTA-SE Molecular Biophysics Research Group, Semmelweis University, Tüzoltó utca 37-47, Budapest H1094, Hungary

$\dagger$ These authors contributed equally to this work.

* Author to whom correspondence should be addressed;

E-Mail: kellermayer.miklos@med.semmelweis-univ.hu; Tel.: +36-1-267-6261; Fax: +36-1-266-6656.

Received: 27 June 2014; in revised form: 29 July 2014 / Accepted: 29 July 2014 /

Published: 4 August 2014

\begin{abstract}
The exclusion zone (EZ) is a boundary region devoid of macromolecules and microscopic particles formed spontaneously in the vicinity of hydrophilic surfaces. The exact mechanisms behind this remarkable phenomenon are still not fully understood and are debated. We measured the short- and long-time-scale kinetics of EZ formation around a Nafion gel embedded in specially designed microfluidic devices. The time-dependent kinetics of EZ formation follow a power law with an exponent of 0.6 that is strikingly close to the value of 0.5 expected for a diffusion-driven process. By using optical tweezers we show that exclusion forces, which are estimated to fall in the sub-pN regime, persist within the fully-developed EZ, suggesting that EZ formation is not a quasi-static but rather an irreversible process. Accordingly, the EZ-forming capacity of the Nafion gel could be exhausted with time, on a scale of hours in the presence of $1 \mathrm{mM} \mathrm{Na}_{2} \mathrm{HPO}_{4}$. EZ formation may thus be a non-equilibrium thermodynamic cross-effect coupled to a diffusion-driven transport process. Such phenomena might be particularly important in the living cell by providing mechanical cues within the complex cytoplasmic environment.
\end{abstract}


Keywords: exclusion zone; microfluidic device; optical trapping; atomic force microscopy; non-equilibrium process; chemical gradient; diffusion; coupled transport

PACS Codes: 05; 47; 64; 68; 82; 83; 97

\section{Introduction}

In the direct vicinity of hydrophilic gel-like materials a zone devoid of microscopic particles, called the exclusion zone (EZ) develops [1]. The size of the EZ may reach several hundred microns, and under some circumstances, it may persist for relatively long times, up to days [2]. The EZ has been shown to develop near the surface of a surprising variety of materials, ranging from myofibrils to hydrophilic lipid monolayers [2]. It has been proposed that EZ formation is caused by the emergence of a special phase of water (termed "EZ water") that displays long-range ordering. This ordering is determined by periodically arranged alternating charges on the gel surface leading to the formation of ordered and undisturbed multilayers of water molecules which eventually arrange into a liquid crystalline phase. Long-range ordering reaching hundreds of microns, however, is unexpected at high temperatures (e.g., room or body temperatures) where thermal effects lead towards structural disordering. Recently, a theory of "macromolecular chemotaxis" has been proposed to explain the emergence of the EZ based on a concentration gradient of cosolutes [3,4]. This theory has been debated [5] and needs experimental testing. Although the EZ has been characterized by numerous experimental approaches [1,6-18], key features related to its thermodynamics and kinetics still need to be investigated. In the present work we measured the short- and long-time-scale kinetics of EZ formation in specially designed microfluidic devices that reduce unwanted local perturbation effects, estimated the magnitude of the exclusion force, and tested key predictions of the most plausible microscopic mechanisms of EZ formation. The experimental observations suggest that EZ formation is best explained as a non-equilibrium thermodynamic cross-effect coupled to a diffusion-driven transport process.

\section{Results and Discussion}

We investigated the mechanisms of formation of an exclusion zone (EZ), a region that becomes devoid of suspended microscopic particles, near the surface of Nafion, a hydrophilic, sulfonated Teflon polymer. Nafion has become, in the recent past, a popular polymeric material in the investigation of EZ properties $[7,15,16,18]$. In order to minimize flow artifacts and to precisely monitor EZ formation, microfluidic devices were constructed that allow the simultaneous, precise control of fluid exchange and time-lapse microscopy (Figure 1). Two types of devices were built, a Parafilm-based device (Figure 1a), and a PDMS-based one (Figure 1b). The results obtained in either of these microfluidic devices were essentially identical. 
Figure 1. Constructs of the microfluidic devices. (a) Schematic diagram of the Parafilm-based microfluidic device; (b) Brightfield microscopic image of the PDMS-based microfluidic device.
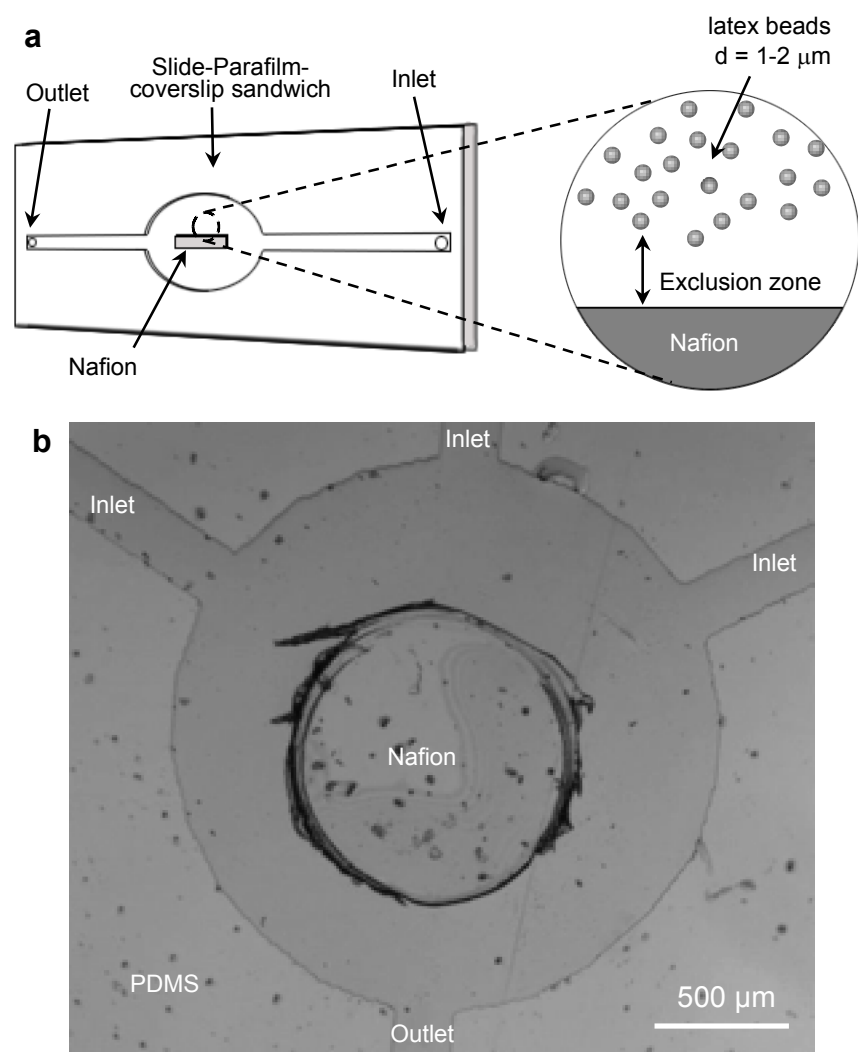

\subsection{Formation of the Exclusion Zone}

Upon the infusion of an aliquot of bead suspension (5\% carboxylated polystyrene beads in MilliQ water) into a microfluidic device containing a freshly prepared piece of Nafion and stopping the fluid flow, a gap progressively formed between the Nafion surface and the bulk of the beads (Figure 2).

Figure 2. Temporal evolution of the exclusion zone. 1- $\mu$ m-diameter carboxylated polystyrene beads $(5 \%)$ suspended in MilliQ water.

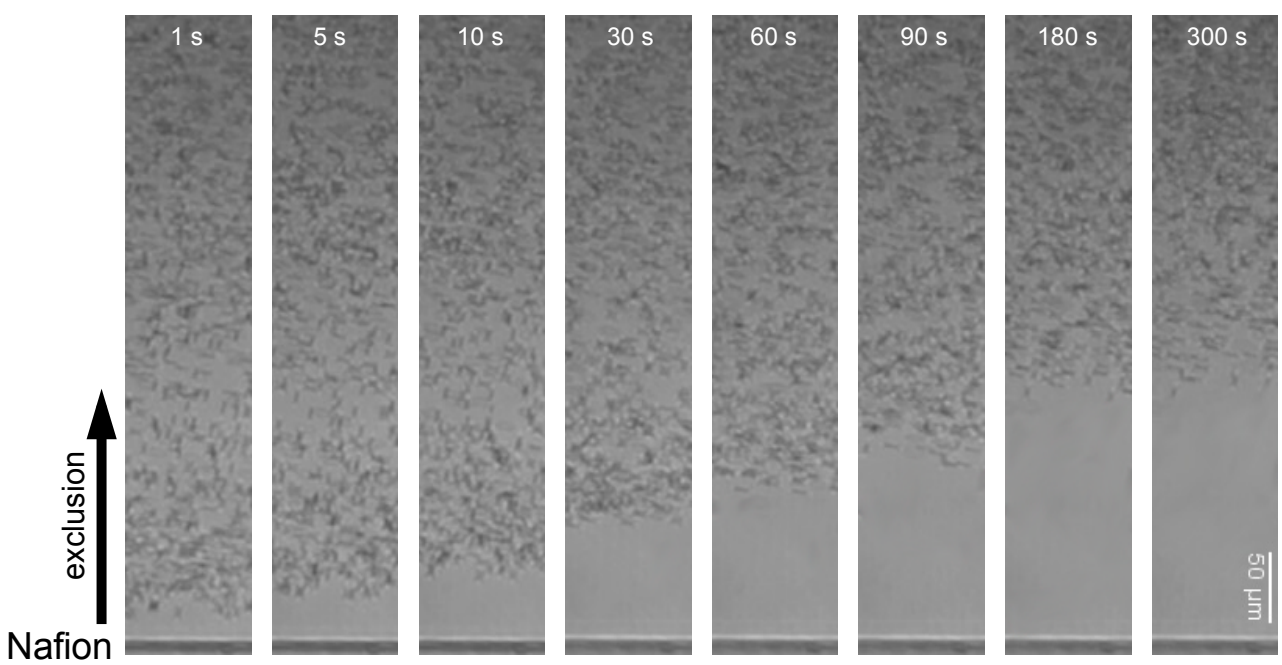


This gap, the size of which far exceeded $100 \mu \mathrm{m}$ (see Figures 3a and 8), corresponds to the exclusion zone. Thus, we confirmed the formation and existence of EZ near the Nafion surface. Although the EZ size reached well above $100 \mu \mathrm{m}$ within a few minutes, its growth did not stop but proceeded with gradually smaller rates (Figure 3a). The size of the EZ $\left(d_{E Z}\right)$ as a function of time $(t)$ followed a power law:

$$
d_{E Z}(t)=A \cdot t^{b}
$$

rather than an exponential function, where $A$ is a prefactor that describes the overall speed of the process (i.e., it corresponds to a phenomenological diffusion coefficient) and $b$ is the exponent that depends on the microscopic mechanisms. We found that, on average, the exponent is 0.6 , which is strikingly close to 0.5 expected for true diffusion [19]. Thus, our time-dependent measurements suggest that EZ formation may be related to a diffusion-driven process. The initial rate of EZ formation allows the estimation of the force $\left(F_{E Z}\right)$ that drives the exclusion process by making a simple assumption about the force balance on a bead (Figure 3a inset):

$$
F_{E Z}+F_{\text {buovant }}-F_{\text {weight }}-F_{\text {Stokes }}=0
$$

where $F_{\text {buoyant }}$ corresponds to the weight of the solvent excluded by a bead $\left(m_{\text {water }} g\right), F_{\text {weight }}$ is the weight of the bead itself ( $m_{\text {bead }} g$ ) and $F_{\text {Stokes }}$ is the hydrodynamic drag force $(6 r \pi \eta v)$ acting on a bead of radius $r$ travelling in a fluid of viscosity $\eta$ with a velocity $v$. Because of the small mass and volume of the beads of both $F_{\text {buoyant }}$ and $F_{\text {weight }}$ may be neglected. This notion is supported by our observation that in a vertically-held microfluidic device the $\mathrm{EZ}$ is symmetrical around (i.e., above and below) the Nafion gel (Figure $3 b$ ), therefore gravity has a negligible effect on the process. Accordingly, $F_{E Z}$ may be calculated as:

$$
F_{E Z} \approx F_{\text {Stokes }}
$$

Considering that the maximal initial exclusion rate in our experiments was $3 \mu \mathrm{m} / \mathrm{s}$ and assuming a viscosity of $1 \mathrm{mPas}$, the initial force that pushes a $2-\mu \mathrm{m}$-diameter bead away from the Nafion surface is calculated as $\sim 60 \mathrm{fN}$. This force $\left(F_{E Z}\right)$ is nearly two orders of magnitude smaller that those measured recently by using optical tweezers [20]. We attribute this discrepancy to an offset of trap calibration caused by the partial interaction of the conical trapping beam with Nafion which leads to unwanted refraction, hence a breakdown of trapping efficiency. The effect of the presence of the Nafion on optical trapping was tested by moving an optically trapped bead towards the surface and measuring the apparent force (Figure 3c). In a similar, control experiment we moved only the laser, with no trapped bead, towards the surface and obtained an identical response (Figure 3d). Our results indicate that near the Nafion surface the force calibration is indeed offset. Because our optical tweezers instrument measures force from changes in photonic momentum [21-23] rather than from bead-based calibration, we may conclude that the offset is indeed due to a local change in refraction. Since the offset exceeds $1 \mathrm{pN}$, measuring the force acting on a bead upon EZ formation is not possible with a precision better than a few piconewtons when using optical tweezers. 
Figure 3. (a) Size of the exclusion zone as a function of time. Datapoints are mean \pm S.E.M. $(n=46)$ from experiments in which different sodium salts (Hofmeister series) of varying concentrations ( $1 \mu \mathrm{M}-10 \mathrm{mM}$ ) were used. Data were fitted with Equation (1). Inset shows the schematic diagram of the instantaneous force balance on the bead; (b) Shape of the exclusion zone in the vicinity of a circular Nafion gel positioned in a vertically mounted PDMS microfluidic device; (c) Apparent force on an optically trapped $3 \mu \mathrm{m}$ bead during its movement towards Nafion surface. Green trace corresponds to the piezo-driven controlled movement of the Nafion towards the optical trap. Red trace corresponds to the force measured by changes in photonic momentum; (d) Control experiment with no bead in the trap.
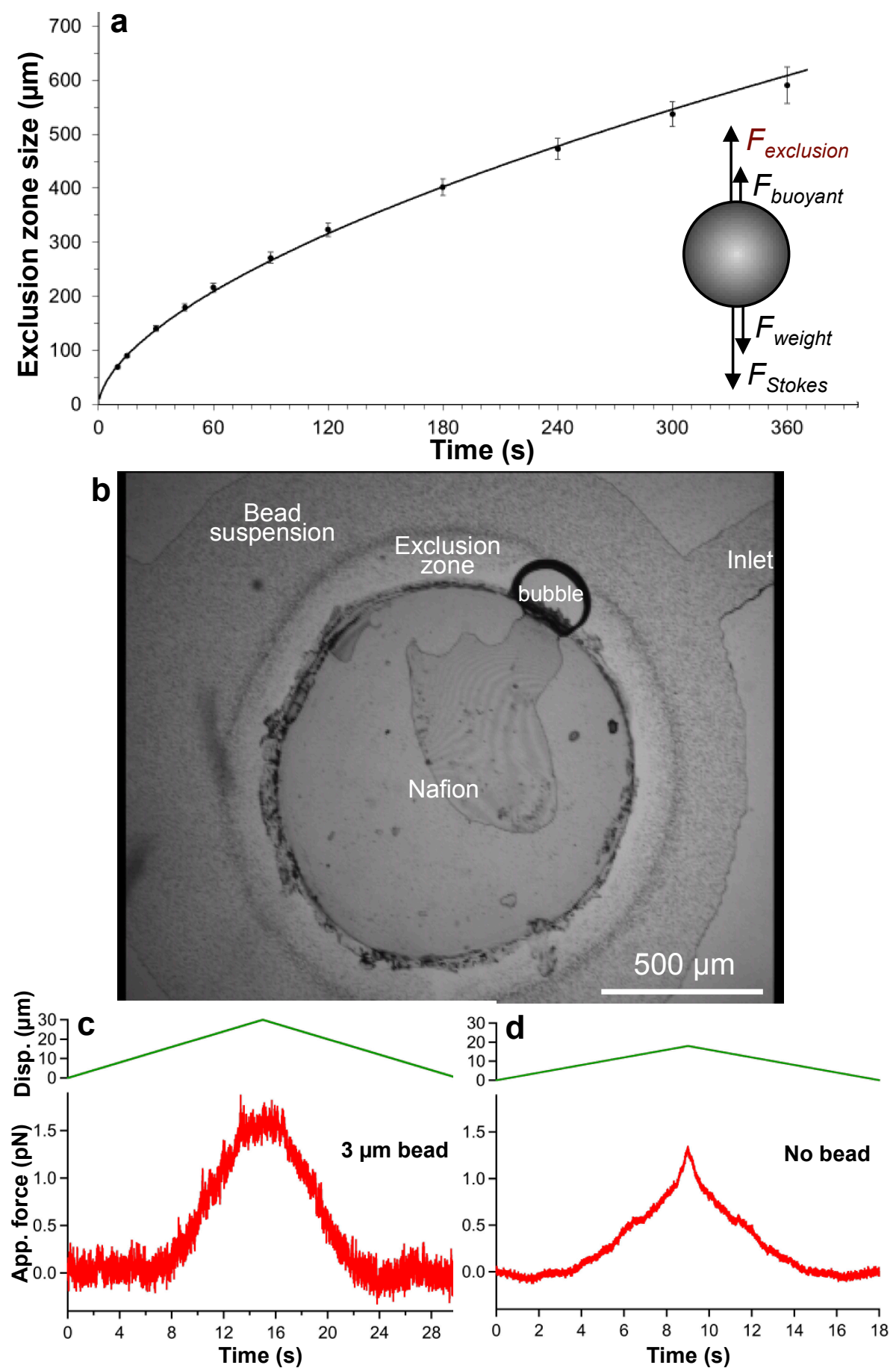


\subsection{Possible Mechanisms of Exclusion Zone Formation}

To distinguish between possible microscopic mechanisms of EZ formation, we assumed that the process may be caused by either: (a) the dissolution of the hydrophilic gel (Figure 4a) during which polymer strands diffusing out of the gel push the beads away from the surface; (b) an entropic brush mechanism (Figure 4b), in which closely spaced, surface-bound, long, elastic polymer strands keep the beads away; (c) the build-up of a distinct phase in an equilibrium process (Figure 4c or 4d) a physicalchemical gradient that sustains a non-equilibrium transport phenomenon (Figure 4d).

Figure 4. Schematics of the tested mechanisms behind EZ formation. (a) Dissolution of the polymer via reptation of polymer strands out of the bulk polymer phase. The reptation channel around a polymer strand is indicated with gray lines. Black arrow indicates the movement of the polymer strand within the reptation channel, red arrow indicates the pushing force acting on the bead; (b) Entropic brush mechanism. Polymer strands (thick black lines) are attached by one end to the polymer surface. The thermally-driven shape fluctuations of the polymer strands result in the generation of force (dotted arrow) that keeps pushing the beads away from the surface; (c) Formation of a distinct phase (equilibrium process). Progressive growth of the phase moves the phase boundary, hence the bulk of the beads, away from the Nafion surface (white arrowhead); (d) Chemical gradient (non-equilibrium process) mechanism. An apparent mechanical force (white arrow) is generated because of the presence of a physical-chemical gradient near the edge of the Nafion surface.
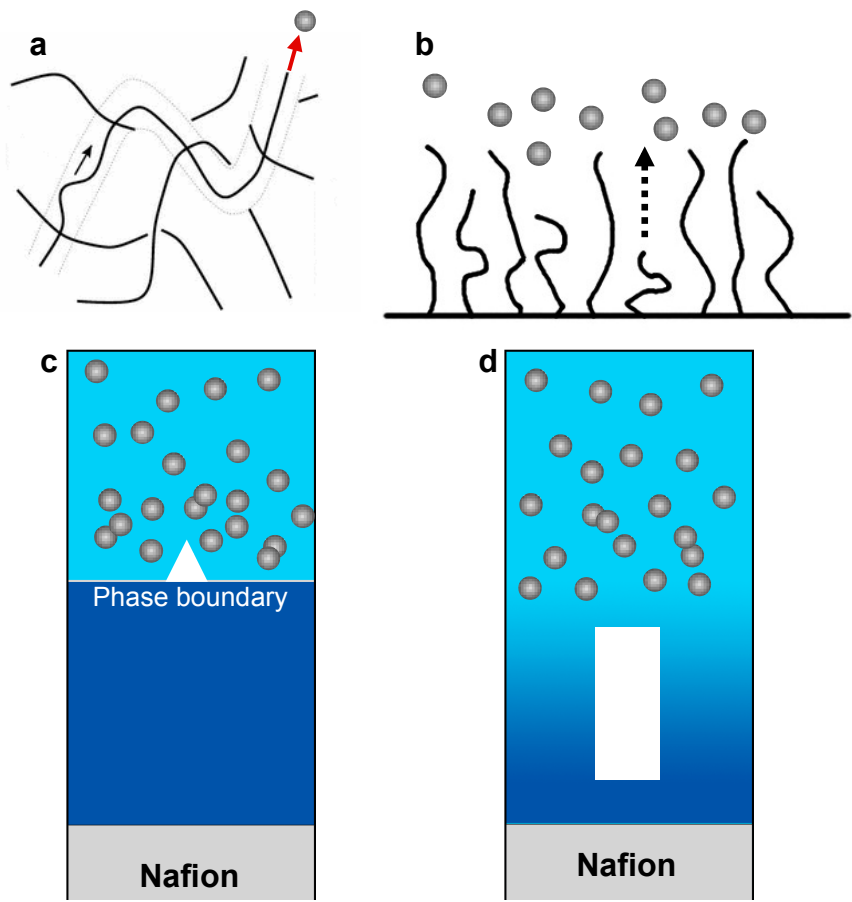

A similar treatise, but with a less distinctive experimental approach has been published before [1], which arrived at the conclusion that the formation of a phase of water with liquid-crystalline properties might be responsible for the EZ (the corresponding mechanism highlighted in Figure 4c). The prediction of the gel dissolution mechanism is that the gel volume eventually diminishes. We found, 
however, that the gel volume remained unchanged even after an eight-hour constant washing with buffer solution (Figure 5). The size, shape and overall appearance of the Nafion disc enclosed in the PDMS-based microfluidic device was identical at the beginning (Figure 5a) and following (Figure 5b) the extended incubation. Furthermore, the gel volume did not change even if the gel was soaked in buffer for several days (data not shown).

Figure 5. Size of the Nafion disc $\left(1 \mathrm{mM} \mathrm{Na}_{2} \mathrm{HPO}_{4}, \mathrm{pH} 7.6\right)$ as a function of time. (a) Bright field microscopic image at the beginning of the experiment $(\mathrm{t}=0)$; (b) Bright field microscopic image recorded after eight hours $(t=8 \mathrm{~h})$.
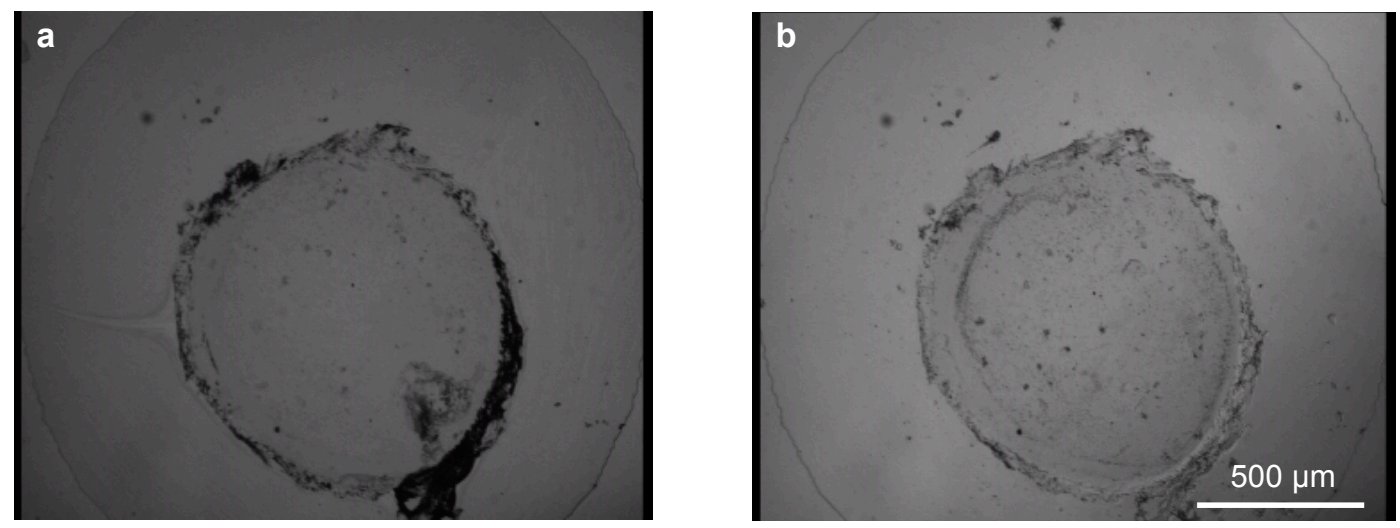

Because the overall structure of the gel appears to remain intact during the EZ-formation process, the gel dissolution mechanism can be excluded. The prediction of the entropic brush mechanism is that polymer chains could be detected, either by structural or mechanical methods, on the gel surface. To investigate the microscopic structure of the Nafion surface, we scanned it by using AFM (Figure 6a). No filamentous structures were observed, and the average surface roughness was less than $1 \mathrm{~nm}$. In addition, during AFM-based force spectroscopic experiments (Figure 6b) we found no mechanical manifestations of any polymer strands that might stand away from the Nafion surface. If an entropic brush was present on the surface, then it is expected to bend the cantilever upward when it approaches the gel surface, resulting in force generation (forces below the baseline in the red trace of Figure $6 b$ ). We found, however, that across a distance with a range of $10 \mu \mathrm{m}$ from the gel surface there was no regime mechanically distinct from the bulk solution (Figure $6 \mathrm{~b}$ red trace). Even if the polymer strands stand so much apart that a mechanically distinct phase is undetectable during surface approach, the polymer chains can be detected by their elastic response when the cantilever is retracted. If polymer strands were present, then a force bending the cantilever downward is expected to appear during retraction (forces above the baseline in the blue trace of Figure $6 \mathrm{~b}$ ). We found, however, that elastic forces were absent in the force trace across a distance of $10 \mu \mathrm{m}$ from the gel surface (Figure $6 \mathrm{~b}$ blue trace). Thus, we exclude the possibility that the EZ is formed by an entropic brush mechanism. To distinguish between a progressive phase formation (Figure 4c) and gradient mechanisms (Figure 4d), optical trapping experiments were carried out (Figure 7 and Supplementary Video 1). During the progressive formation of a distinct phase it is the moving phase boundary that pushes the beads away from the gel surface. Accordingly, a bead trapped within the phase is unlikely to be exposed to a net force. By contrast, if a gradient prevails, a bead trapped within the EZ remains exposed to forces. Trapping a bead within the exclusion zone without directly and significantly interfering with the 
overall mechanics of EZ formation (i.e., with the moving EZ boundary) can be carried out with optical tweezers. The force acting on this trapped particle can then be tested by releasing it from the optical trap (turning off the laser) and investigating its motion.

Figure 6. Investigation of the Nafion surface with AFM. (a) Height-mode AFM image of a Nafion surface in distilled water; (b) Force spectrum recorded by tapping the Nafion surface upon approach (red trace) from bulk solution (MilliQ water) and retraction (blue trace) from the surface.

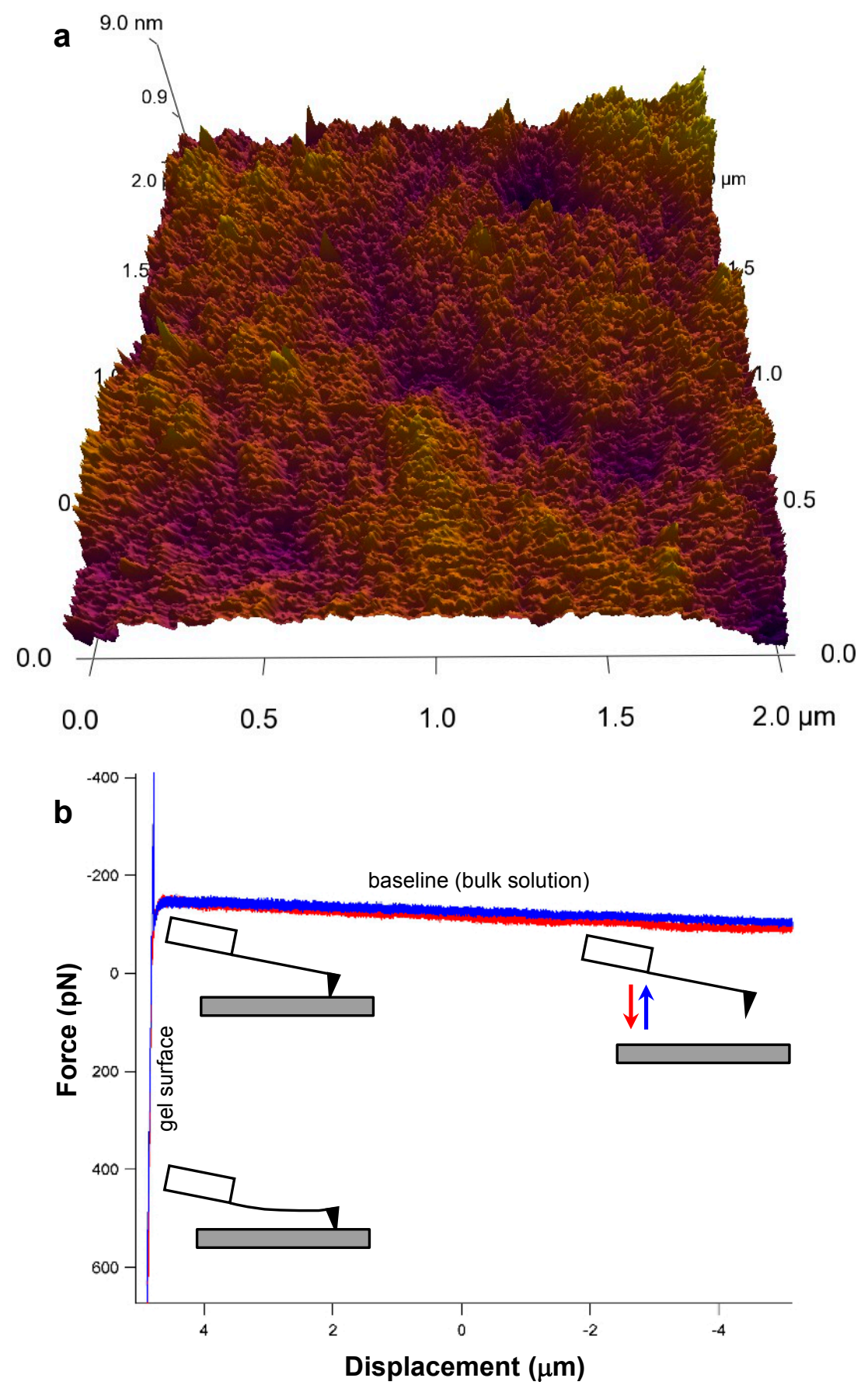


The prediction of the moving boundary mechanism is that, upon release, the bead within the exclusion zone stays in its average position. By contrast, if a gradient is present, then the bead is expected to move in the direction of the EZ formation, driven by the gradient force. The results are shown in Figure 7 (see also Supplementary Video 1). A few beads were trapped $50 \mu \mathrm{m}$ away from the Nafion surface. Notably, the presence of the optical trap did not cause any perturbation in the distribution of the beads in the vicinity, suggesting that exposure to the trapping laser did not cause significant effects in EZ formation. Once the boundary of the EZ had passed the position of the trapped beads and reached a distance of $100 \mu \mathrm{m}$ away from the Nafion surface, the trapping laser was switched off to test the effect of net forces on the beads left within the already established EZ. It was observed that many of the beads (except the ones stuck to the glass side of the microfluidic device) started moving away from the Nafion surface and soon caught up with the beads in the bulk phase. Thus, net forces are present within the EZ, which therefore cannot be considered a homogenous phase. Our results contradict the moving phase boundary mechanism and favor the idea that the EZ is generated and sustained by physical-chemical gradient. The conclusion drawn from the simple experiment described above is further supported by the findings of recent optical trapping measurements by Chen et al. [20] who demonstrated that net forces act on a bead held within the EZ. Although there is uncertainty about the magnitude of the forces because of local refraction and calibration issues (see above), there is no doubt about the presence of a net force.

Figure 7. Optical trapping of beads in the vicinity of Nafion. See also Supplementary Video 1. Red arrow shows the location of the optical trap. White arrows show the beads moving after turning off the trapping laser. Some of the beads remained at the trap position due to sticking to the glass wall of the microfluidic device. We observed this when moving the stage after the experiment, which moved the surface-stuck beads in accordance (data not shown).
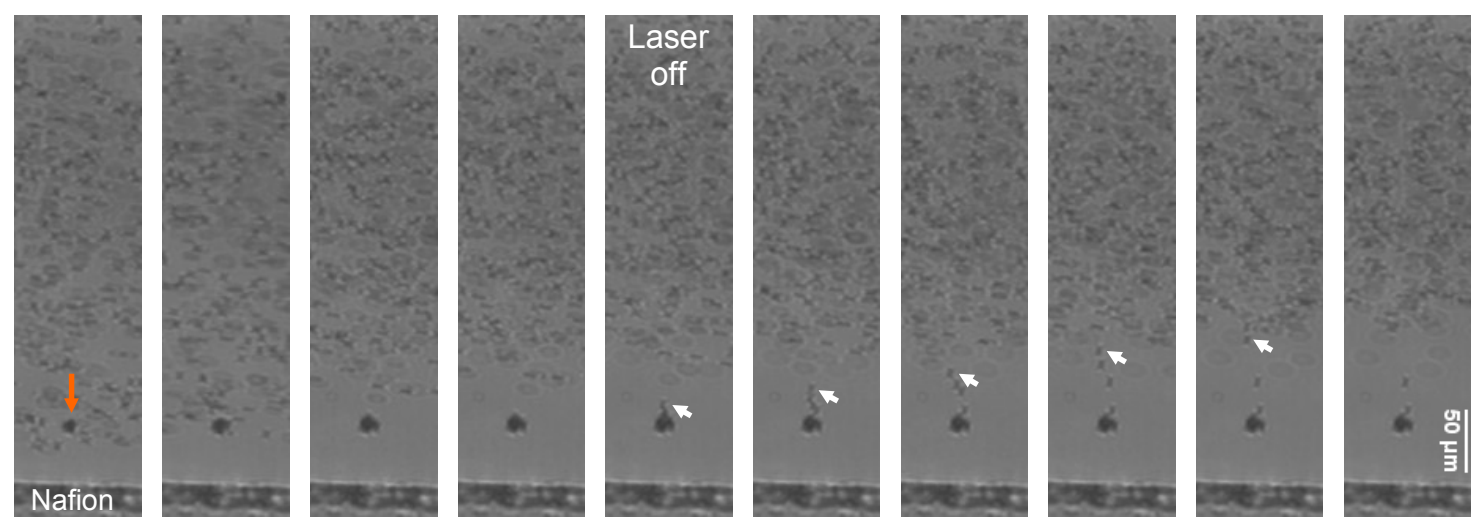

\subsection{Kinetics of Exclusion Zone Formation}

If a physical-chemical gradient is responsible for the formation of the exclusion zone, then the EZ is expected to vanish with time once the gradient, which sustains the non-equilibrium transport process, equilibrates. To test the temporal persistence of the EZ, time-dependent measurements which lasted up to several days were performed. The experimental results are shown in Figure 8. Throughout the experiment a Nafion disc was continuously washed with a buffer solution $\left(1 \mathrm{mM} \mathrm{Na}_{2} \mathrm{HPO}_{4}\right)$. 
Figure 8. Kinetics of EZ formation as a function of incubation time (shown in the upper left corners of each image). See also Supplementary Videos 2-4. Kymograms (images of distance vs. time) were recorded along a radial segment between the Nafion and PDMS edges. Red curve indicates the temporal change in the edge of the bead suspension, which corresponds to the EZ size.

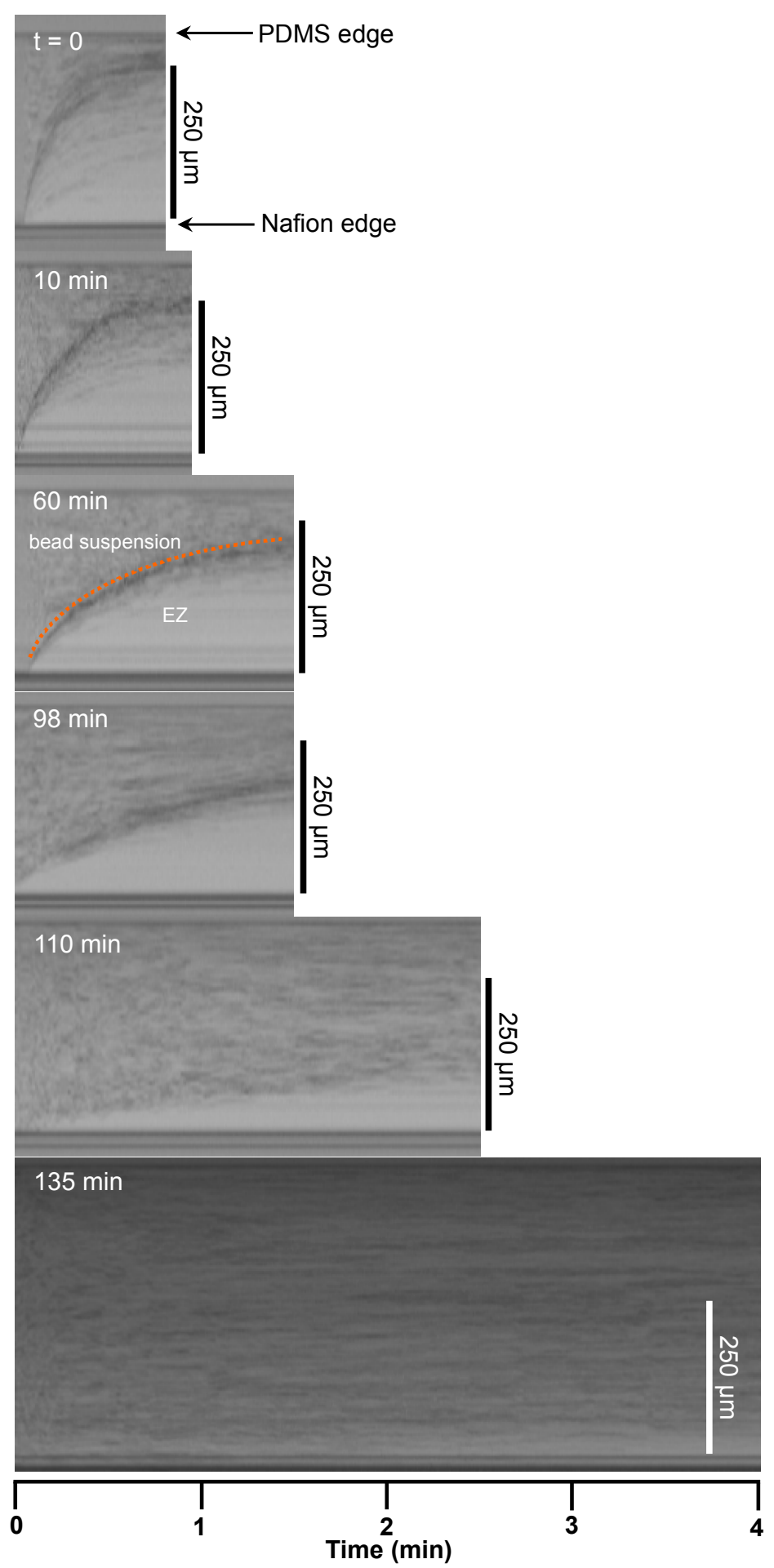

At certain time points an aliquot of bead suspension wash flushed in the microfluidic device to probe whether an EZ can be formed. If a static distribution of surface charges is responsible for EZ formation, then the EZ is expected to form regardless of how long the gel was incubated. If, however, 
a transport process is responsible, then the EZ is re-established only as long as the gradient (e.g., difference in $\mathrm{H}^{+}$concentration between the interior of Nafion and the bulk phase) sustaining the transport is present. At the beginning of the experiment (Figure 8, Supplementary Video 2), 1- $\mu$ m-diameter carboxylated polystyrene beads suspended in a buffer solution of $1 \mathrm{mM} \mathrm{Na}_{2} \mathrm{HPO}_{4}$ were rapidly (within $1 \mathrm{~min}$ ) excluded from the vicinity of Nafion. After one hour the exclusion rate was significantly reduced (Supplementary Video 3), and after $110 \mathrm{~min}$ EZ formation was hardly detectable (Supplementary Video 4). After an overnight washing of the Nafion gel EZ formation completely vanished. Thus, EZ formation can be exhausted, indicating that the process is indeed a non-equilibrium transport phenomenon driven by a physical-chemical gradient. Once this gradient equilibrates, the driving force of EZ formation vanishes and the phenomenon can no longer be evoked.

The source of the gradient in our experiment was most likely the large quantity of $\mathrm{H}^{+}$ions present in the Nafion gel at the beginning of the experiment. $\mathrm{H}^{+}$ions are transported, by diffusion, from the gel into the surrounding medium along the concentration gradient which vanished here within a couple hours. Interestingly, and in contrast to this result, it has been claimed that the EZ may persist for days [2]. We currently hypothesize that if no exchangeable ions are present in the bulk solution, then the transport of $\mathrm{H}^{+}$ions is slow. Therefore, a long time is required for the gradient to disappear completely. If, however, exchangeable ions such as $\mathrm{Na}^{+}$of the $\mathrm{Na}_{2} \mathrm{HPO}_{4}$ buffer solution are present, then the process is highly facilitated. In support of this hypothesis we observed that the formation of the EZ around a fresh Nafion gel in $1 \mathrm{mM} \mathrm{Na}_{2} \mathrm{HPO}_{4}$ is a fast process that proceeds on the time scale of seconds (Figure 8, $\mathrm{t}=0$ ). By contrast, if deionized (MilliQ) water was present instead of the buffer solution, EZ formation slowed down considerably and proceeded on a time scale of minutes (Figure 2). Further preliminary experiments performed by the authors indicate that the type and concentration of the exchangeable ions also influence the rate of EZ formation and are likely to affect its persistence as well [24].

The data presented in this work suggest that the flux of beads away from the gel surface, hence the formation of an exclusion zone is a non-equilibrium phenomenon coupled to diffusion-driven material transport rather than the quasi-static build-up of a highly ordered water phase [2]. EZ formation may therefore be related to the Soret and Dufour effects which are coupled thermodynamic effects between irreversible processes driven by temperature and concentration gradients, respectively [25]. The possibility that a concentration gradient may lead to generation of mechanical forces causing the remarkable macroscopic displacement of particles is a novelty and has been addressed recently as "macromolecular chemotaxis" [3]. Although this idea is debated [5] and needs experimental testing, the results presented here indicate that it might provide a plausible explanation for EZ formation. Coupled non-equilibrium transport processes leading to mechanical force generation may be important in the living cell. Here local gradients prevail in an environment packed with macromolecules and organelles with sizes comparable to those of the excluded beads. Although meager if compared with the stall forces of various motor proteins $[19,26]$, EZ force may assist motile and transport processes by providing a reference force field and mechanical cues within the complex cellular environment. 


\section{Experimental Section}

\subsection{General Information}

All experiments were carried out at room temperature. Chemicals were of reagent grade and obtained from Sigma-Aldrich Chemical, Co. (St. Louis, MO, USA). The Nafion gel used was the 50- $\mu$-thick NRE-212 perfluorinated membrane (Sigma-Aldrich, Co.). MilliQ water (Millipore, Darmstadt, Germany) with specific resistance exceeding $18 \mathrm{M} \Omega \mathrm{cm}$ was used throughout the experiments.

\subsection{Parafilm-Based Microfluidic Device}

The Parafilm-based device is a sandwich composed of a microscope slide and coverslip $(60 \mathrm{~mm} \times 24 \mathrm{~mm}$, No. 1) with a layer of Parafilm (100 $\mu \mathrm{m}$ thick) in between (Figure 1a). Two $1 \mathrm{~mm}$ holes were tapped with diamond bits in the slide which served as inlet and outlet, respectively. The slide and coverslip were cleaned in three consecutive 20-minute-long cycles of sonication in acetone, ethanol and MilliQ water, then dried in a stream of high-purity $\mathrm{N}_{2}$ gas. A flow-through channel was engraved into a $60 \mathrm{~mm} \times 24 \mathrm{~mm}$ Parafilm with an Epilog Mini 18 laser engraver (Epilog Laser, Golden, $\mathrm{CO}, \mathrm{USA}$ ) so as to form a reactor (total volume $5 \mu \mathrm{L}$ ) that houses the Nafion gel. A $5 \mathrm{~mm} \times 6 \mathrm{~mm}$ stripe of Nafion membrane folded in half along the shorter edge was placed in the reactor. The device was sealed by gentle heating of the Parafilm layer on a hotplate. The device was then mounted in a bracket with silicon-rubber tubing connectors pressed against the inlets and outlets for access to fluid-flow control.

\subsection{PDMS-Based Microfluidic Device}

The PDMS-based microfluidic device is a microfabricated channel and reactor system mounted on a microscope slide or coverslip (60 $\mathrm{mm} \times 24 \mathrm{~mm}$ ) (Figure $1 \mathrm{~b}$ ). Channel layouts were designed using AutoCAD 2013 (Autodesk, Inc., San Rafael, CA, USA) and devices were produced using standard microfabrication soft-lithographic techniques [27,28] by casting polydimethylsiloxane (PDMS, Sylgard ${ }^{\circledR}$ 184, Dow Corning, Midland, MI, USA.) on a SU-8 (MicroChem, Newton, MA, USA) positive relief patterned mold on a 4 " silicon wafer produced by photolithography. PDMS monomer and curing agent were mixed at a ratio of 10:1 (v/v), degassed and poured over the master and set aside at $70{ }^{\circ} \mathrm{C}$ for $2 \mathrm{~h}$ for crosslinking. The liquid PDMS pre-polymer conforms to the shape of the master and replicates the features of the master. The crosslinked PDMS was removed from the mold and $0.75 \mathrm{~mm}$ inlet and outlet ports were fabricated through the PDMS slab using a Harris Uni-Core biopsy punch (Ted Pella, Inc., Redding, CA, USA). Channel height was 50 microns to conform with the thickness of Nafion membranes. The total reactor volume was $1.5 \mu \mathrm{L}$. Predefined size Nafion membranes were cut and placed into the microfluidic channel immediately following surface treatment. The patterned PDMS slab was bonded to microscope glass slide following surface treatment by Plasma-preen II 863 (Plasmatic Systems, Inc., North Brunswick, NJ, USA). Finally, polyethylene tubes were inserted into the access holes for fluidic contacts. 


\subsection{Microfluidic Experiment and Exclusion-Zone Observation}

Either of the microfluidic devices was mounted vertically on the piezoelectric stage (Thorlabs, Newton, NJ, USA) of a custom-built optical tweezers apparatus coupled with a video-microscopic system. Pressure-driven flow control was achieved by using computer-driven solenoid valves which connected a pressurized buffer-filled bottle to sources of high air pressure and vacuum. Flow rates varied between $0.01 \mathrm{~mL} / \mathrm{h}$ and $0.5 \mathrm{~mL} / \mathrm{h}$. An aliquot $(<50 \mu \mathrm{L})$ of $1 \mu \mathrm{m}$ carboxylated polysterene beads (Polysciences, Warrington, PA, USA) suspended in either MilliQ water or buffer solution (1 $\mathrm{mM}$ $\mathrm{Na}_{2} \mathrm{HPO}_{4}, \mathrm{pH}$ 7.6) to a final concentration of $2.5 \%-5 \%$, was injected into and flown through the microfluidic device with a syringe. Subsequently, the device was washed with MilliQ water or buffer solution by using the flow control system. During EZ formation both the inlet and outlet tubes were clamped so as to stop flow as completely as possible. The stage was illuminated with a blue LED source (470 nm, $60 \mathrm{~mW}$, Thorlabs). At this setting the illuminance in the plane of the sample was $300 \mathrm{lux}$, which was unaffected regardless of whether the ambient lighting was turned on or off. The sample and visualized with $3.2 \times$ or $10 \times$ objective lenses (Zeiss, Oberkochen, Germany) by using an analog grayscale CCD camera (Sony, Tokyo, Japan). Images were recorded digitally at a rate of 25 frames/s.

\subsection{Optical Tweezers}

Manipulation of polystyrene beads was carried out with a custom-built dual-beam counter-propagating optical tweezers apparatus [21-23]. For force measurements the optical trap was formed with two 60× 1.2 NA water immersion objective lenses (Olympus, Tokyo, Japan) mounted on opposite sides of a Parafilm-based microfluidic device with coverslip walls. The light sources were $833 \mathrm{~nm} 200 \mathrm{~mW}$ single-mode diode lasers (SDL 5400 series). Trap stiffness was $\sim 0.2 \mathrm{pN} / \mathrm{nm}$. For imaging trapped beads within the EZ in a large field of view, only one water immersion objective lens was used, and the microfluidic device was imaged with a $3.2 \times$ objective from the opposite side. Instrument control and data acquisition were managed by using custom-written LabView routines.

\subsection{Atomic Force Microscopy and Force Spectroscopy}

The surface of the hydrated Nafion gel was imaged with a high-resolution atomic force microscope (MFP3D, AsylumResearch, Santa Barbara, CA, USA). $512 \times 512$ images were collected in tapping mode with a stiff cantilever (AC160, Olympus) at a pixel resolution of $4 \mathrm{~nm}$. For force spectroscopic measurements soft cantilevers (MHW-AUCT, Veeco, Inc., Plainview, New York, NY, USA) calibrated for stiffness with the thermal method [29] were used. The entire vertical scanning range $(10 \mu \mathrm{m})$ of the AFM instrument was used in the force spectroscopic measurements so as to screen as large part of the EZ as possible.

\subsection{Data Analysis}

Video microscopic images were captured and analyzed with the ImageJ program (version 1.48, public domain). Distance measurements were carried out on the images with the built-in tools of the program. For spatial calibration, stage micrometers (10 $\mu \mathrm{m}$ division) were used for each objective lens. AFM images were analyzed with the software module of the AsylumResearch MFP3D instrument. 
Numerical data were displayed, analyzed and fitted by using IgorPro (version 6.0, Wavemetrics, Lake Oswego, OR, USA).

\section{Conclusions}

The short-time-scale kinetics of EZ formation follow a power law with an exponent of 0.6. Because this exponent is very close to the 0.5 value expected for free diffusion, EZ formation is most likely driven by or coupled to a diffusion-based process. From the initial rates of EZ formation we estimate that the force pushing a microscopic bead away from the Nafion surface is well below the piconewton regime. Because beads optically trapped within the EZ keep being pushed away from Nafion once the trap is turned off, forces prevail within the EZ which is unexpected for a quasi-static phase of ordered water molecules. Rather, the forces emerge from a diffusive transport process sustained by a gradient between the Nafion and the bulk solution. In support of this notion, removing the gradient by extensively washing the Nafion gel with buffer completely exhausted its EZ-forming capacity within a couple of hours. Thus, EZ formation appears to be a non-equilibrium thermodynamic cross-effect coupled to a diffusion-driven transport, which remarkably manifests in the generation of mechanical forces. Although these forces are rather small, they may provide mechanical cues within a complex macromolecular environment such as the interior of the living cell.

\section{Acknowledgments}

This work was supported by grants from the Hungarian Science Foundation (OTKA K84133, OTKA K109480). The research leading to these results has received funding from the European Union's Seventh Framework Program (FP7/2007-2013) under grant agreement $n^{\circ}$ HEALTH-F2-2011278850 (INMiND).

\section{Author Contributions}

István N. Huszár designed, performed and analyzed experiments, wrote manuscript. Zsolt Mártonfalvi designed, performed and analyzed experiments. András József Laki and Kristóf Iván designed and provided the PDMS-based microfluidic device. Miklós Kellermayer designed, performed and analyzed experiments, in addition to writing the manuscript. All authors have read and approved the final published manuscript.

\section{Conflicts of Interest}

The authors declare no conflict of interest.

\section{References}

1. Zheng, J.M.; Pollack, G.H. Long-range forces extending from polymer-gel surfaces. Phys. Rev. E 2003, 68, 031408.

2. Pollack, G. The Fourth Phase of Water; Ebner and Sons Publishers: Seattle, WA, USA, 2013.

3. Schurr, J.M.; Fujimoto, B.S.; Huynh, L.; Chiu, D.T. A Theory of Macromolecular Chemotaxis. J. Phys. Chem. B 2013, 117, 7626-7652. 
4. Schurr, J.M. Phenomena associated with gel-water interfaces. Analyses and alternatives to the long-range ordered water hypothesis. J. Phys. Chem. B 2013, 117, 7653-7674.

5. Pollack, G.H. Comment on "A theory of macromolecular chemotaxis" and "Phenomena associated with gel-water interfaces. Analyses and alternatives to the long-range ordered water hypothesis". J. Phys. Chem. B 2013, 117, 7843-7846.

6. Tanchak, O.M.; Yager, K.G.; Fritzsche, H.; Harroun, T.; Katsaras, J.; Barrett, C.J. Water distribution in multilayers of weak polyelectrolytes. Langmuir 2006, 22, 5137-5143.

7. Zheng, J.M.; Chin, W.C.; Khijniak, E.; Khijniak, E.; Pollack, G.H. Surfaces and interfacial water: evidence that hydrophilic surfaces have long-range impact. Adv. Colloid Interface Sci. 2006, 127, $19-27$.

8. Klimov, A.; Pollack, G.H. Visualization of charge-carrier propagation in water. Langmuir 2007, 23, 11890-11895.

9. Zhao, Q.; Zheng, J.; Chai, B.; Pollack, G.H. Unexpected effect of light on colloidal crystal spacing. Langmuir 2008, 24, 1750-1755.

10. Chai, B.H.; Zheng, J.M.; Zhao, Q.; Pollack, G.H. Spectroscopic studies of solutes in aqueous solution. J. Phys. Chem. A 2008, 112, 2242-2247.

11. Zheng, J.M.; Wexler, A.; Pollack, G.H. Effect of buffers on aqueous solute-exclusion zones around ion-exchange resins. J. Colloid Interface Sci. 2009, 332, 511-514.

12. Chai, B.; Yoo, H.; Pollack, G.H. Effect of radiant energy on near-surface water. J. Phys. Chem. B 2009, 113, 13953-13958.

13. Chai, B.; Pollack, G.H. Solute-free interfacial zones in polar liquids. J. Phys. Chem. B 2010, 114, 5371-5375.

14. Yoo, H.; Paranji, R.; Pollack, G.H. Impact of Hydrophilic Surfaces on Interfacial Water Dynamics Probed with NMR Spectroscopy. J. Phys. Chem. Lett. 2011, 2, 532-536.

15. O'Rourke, C.; Klyuzhin, I.; Park, J.S.; Pollack, G.H. Unexpected water flow through Nafion-tube punctures. Phys. Rev. E 2011, 83, 056305.

16. Figueroa, X.A.; Pollack, G.H. Exclusion-Zone Formation From Discontinuous Nafion Surfaces. Int. J. Des. Nat. Ecodyn. 2011, 6, 286-296.

17. Chai, B.; Mahtani, A.G.; Pollack, G.H. Unexpected Presence of Solute-Free Zones at Metal-Water Interfaces. Contemp. Mater. 2012, 3, 1-12.

18. Das, R.; Pollack, G.H. Charge-based forces at the Nafion-water interface. Langmuir 2013, 29, 2651-2658.

19. Phillips, R.; Kondev, J.; Theriot, J. Physical Biology of the Cell; Garland Science: New York, NY, USA, 2009.

20. Chen, C.S.; Chung, W.J.; Hsu, I.C.; Wu, C.M.; Chin, W.C. Force field measurements within the exclusion zone of water. J. Biol. Phys. 2012, 38, 113-120.

21. Bianco, P.; Nagy, A.; Kengyel, A.; Szatmári, D.; Mártonfalvi, Z.; Huber, T.; Kellermayer, M.S. Interaction forces between F-actin and titin PEVK domain measured with optical tweezers. Biophys. J. 2007, 93, 2102-2109.

22. Kellermayer, M.S.; Smith, S.B.; Granzier, H.L.; Bustamante, C. Folding-unfolding transitions in single titin molecules characterized with laser tweezers. Science 1997, 276, 1112-1116. 
23. Mártonfalvi, Z.; Bianco, P.; Linari, M.; Caremani, M.; Nagy, A.; Lombardi, V.; Kellermayer, M. Low-force transitions in single titin molecules reflect a memory of contractile history. J. Cell Sci. 2014, 127, 858-870.

24. Huszár, I.; Mártonfalvi, Z.; Kellermayer, M. Effect of Hofmeister-series salts on the dynamics of exclusion-zone formation. 2014, manuscript in preparation.

25. Mortimer, R.G.; Eyring, H. Elementary transition state theory of the Soret and Dufour effects. Proc. Natl. Acad. Sci. USA 1980, 77, 1728-1731.

26. Howard, J. Mechanics of Motor Proteins and the Cytoskeleton; Sinauer Associates, Inc.: Sunderland, MA, USA, 2001.

27. Duffy, D.C.; McDonald, J.C.; Schueller, O.J.; Whitesides, G.M. Rapid Prototyping of Microfluidic Systems in Poly (dimethylsiloxane). Anal. Chem. 1998, 70, 4974-4984.

28. McDonald, J.C.; Whitesides, G.M. Poly (dimethylsiloxane) as a material for fabricating microfluidic devices. Acc. Chem. Res. 2002, 35, 491-499.

29. Hutter, J.; Bechhoefer, J. Calibration of atomic-force microscope tips. Rev. Sci. Instrum. 1993, 64, $1868-1873$.

(C) 2014 by the authors; licensee MDPI, Basel, Switzerland. This article is an open access article distributed under the terms and conditions of the Creative Commons Attribution license (http://creativecommons.org/licenses/by/3.0/). 